\title{
Breast reconstruction with tissue expansion followed by serial autologous fat transfer: a report of six cases
}

\author{
Chen Zhang, MD, $\mathrm{PhD}^{1,2}$, Xiaoli Jiang, $\mathrm{MMs}^{3}$, Linan Song, $\mathrm{BMs}^{4}$, Chunying $\mathrm{Ge}, \mathrm{MD}^{3}$ \\ ${ }^{1}$ Institute of Plastic Surgery of Dalian University, Dalian, P.R. China, ${ }^{2}$ Shenyang Mylike Aesthetic Hospital, Shenyang, P.R. China, ${ }^{3}$ New York \\ Fashion Cosmetic Clinic, Dalian, P.R. China, ${ }^{4}$ Aid BCC Clinic, Dalian, P.R. China
}

\begin{abstract}
To introduce a new method of breast reconstruction using tissue expander followed by serial autologous fat transfer (AFT). From May 1, 2013, to April 30, 2016, six breasts from six female patients (age range, 35-62 years) were reconstructed using tissue expansion followed by serial AFT. Clinical data were obtained, and each step of the operative methods was recorded. All six patients presented satisfactory symmetry in the contralateral breast. Three AFTs were performed in one patient, whereas four and five AFTs were performed in one patient and four patients, respectively. The intervals between the two adjacent AFTs were 3-5 months. A 450-ml round expander was used in this series, and the volume of each AFT was 50-150 ml. Two patients underwent mastopexy on the contralateral breasts to correct severe breast ptosis. Two patients underwent nipple-areola complex reconstruction 6 months after the last AFT. Tissue expansion followed by serial AFT is a safe and effective method for breast reconstruction. It can be an option for patients who do not prefer pedicled TRAM or DIEP flap transfer or pedicled latissimus dorsi myocutaneous flap coverage with implant placement and those who cannot afford the Brava plus fat graft.
\end{abstract}

Keywords: fat graft; tissue expansion; breast reconstruction

\section{Introduction}

Autologous fat transfer (AFT) can be used for breast augmentation, correction of breast asymmetry and deformities, breast reconstruction as a primary and adjunct technique, and soft tissue coverage of breast implants [1]. However, in some patients, the remnant mastectomical skin and soft tissue are very thin and adhere to the underlying muscles or ribs. In this case, the soft tissue space for AFT is not sufficient; hence, the golden standard methods for breast reconstruction are pedicled TRAM or DIEP flap transfer or pedicled latissimus dorsi myocutaneous flap coverage with implant placement [2]. However, patients may refuse the use of any flap transfer because it might result in obvious scarring, secondary damage to the donor site, and longterm recovery. Khouri et al.[3] invented Brava, and showed that adding Brava expansion before autologous fat grafting leads to significantly larger breast augmentations, with more fat graft placement, higher graft survival rates, and minimal graft necrosis or complications, demonstrating high safety and efficacy for the procedure. Moreover, Mestak et al. [4], Khouri et al. [5], and Hammer-Hansen et al. [6] reported that adding Brava to breast reconstructions can expand the skin and soft tissue for AFT after traditional or modified mastectomy. Nevertheless, if patients cannot afford Brava and insist that breast reconstruction with 
AFT be performed, what options do plastic surgeons have? Is it feasible to perform breast reconstruction with tissue expansion followed by serial AFT?

\section{Clinical materials}

\section{Patient data}

From May 1, 2013, to April 30, 2016, six breasts from six female patients (age range, 35-62 years) were reconstructed using tissue expansion followed by serial AFT. The principles outlined in the Declaration of Helsinki were followed before this new method of breast reconstruction was performed. The patients provided informed consent. The breast reconstructions were performed 3 months to 10 years after mastectomy without irradiation for breast cancer. The patients were followed up for at least 1 year (Table 1$)$.

\section{Surgical techniques}

\section{Tissue expander placement}

The 4-cm incision was marked at the middle part of the mastectomy scar. A pocket was subsequently undermined beneath the pectoralis major. Its upper, medial, and lateral margins were similar to those of silicone implant for augmentation mammoplasty. However, its lower margin depended on the status of the contralateral breast. If the status was normal, the lower margin of the expander pocket should be $1-2 \mathrm{~cm}$ beyond the contralateral inframammary fold. If mild and moderate or severe breast ptoses were noted, the lower margin of the expander pocket should be $2-3 \mathrm{~cm}$ and $3-4 \mathrm{~cm}$ lower than the contralateral inframammary fold, respectively. Subsequently, the expander, filled with a 300-ml antibiotic saline, was placed beneath the pectoralis major, generally without skin tension. The injecting port was pulled out of the pocket through the incision at the mastectomy draining scar and kept out of the body until the expander was removed (Fig. 1A).

\section{Serial AFT into the soft tissue and pectoralis major}

During expander insertion, fat was simultaneously harvested from the abdomen and transferred into the soft tissue and pectoralis major on the mastectomy side using the Coleman technique [1]. In this technique, a 10-ml syringe was used to aspirate the fat under negative pressure. The fat was centrifuged at 3,000 rpm for $5 \mathrm{~min}$ to separate the fat from the blood and the remains of fatty acids. Subsequently, the fat was transferred into $1 \mathrm{ml}$ syringes for grafting. After the expander was placed and filled into the pocket, the fat grafts were injected into the subcutaneous space and pectoralis major with a $1.2 \mathrm{G}$ blunt cannula through multiple tunnels.

Besides the first AFT accompanying the tissue expander insertion, another 2-4 AFTs will be performed at 3-6 month intervals. The interval between the placement of tissue expander and the first AFT was at least 5 months. The fat was harvested using the methods mentioned above during each AFT. The expander was deflated by withdrawing $50-150 \mathrm{ml}$ saline after the fat was harvested. Subsequently, the same amount of fat was injected into the subcutaneous space and pectoralis major. All saline in the expander was withdrawn during the last surgery and during the reconstruction of the new breast (Fig. 1B, Fig. 1C).

\section{Nipple-areola complex reconstruction}

Nipple-areola complex (NAC) reconstruction was performed 6 months after the last AFT. The NAC donor was from the contralateral NAC. The nipple of the normal side was cut in half from its center. If the diameter of the areola was $>4 \mathrm{~cm}$, the

Table 1. Patients summaries for breast reconstruction with TE followed by serial AFT

\begin{tabular}{|c|c|c|c|c|c|c|c|}
\hline No & Age & Size of TE & Amount of NS & Times of AFT & Volume of AFT $\times$ Times & Mastopexy & Reconstrution of NAC \\
\hline 1 & 62 & 450 & 500 & 5 & $100 \times 350 \times 2$ & $\mathrm{Y}$ & $\mathrm{Y}$ \\
\hline \multirow[t]{2}{*}{2} & 41 & 450 & 450 & 3 & $150 \times 1$ & $\mathrm{~N}$ & $\mathrm{Y}$ \\
\hline & & & & & $100 \times 2$ & & \\
\hline 3 & 45 & 450 & 500 & 4 & $100 \times 350 \times 1$ & $\mathrm{Y}$ & $\mathrm{N}$ \\
\hline 4 & 35 & 450 & 450 & 5 & $100 \times 450 \times 1$ & $\mathrm{~N}$ & $\mathrm{~N}$ \\
\hline 5 & 58 & 450 & 450 & 5 & $100 \times 450 \times 1$ & $\mathrm{~N}$ & $\mathrm{~N}$ \\
\hline \multirow[t]{3}{*}{6} & 48 & 450 & 550 & 5 & $150 \times 1$ & $\mathrm{~N}$ & $\mathrm{~N}$ \\
\hline & & & & & $100 \times 3$ & & \\
\hline & & & & & $50 \times 1$ & & \\
\hline
\end{tabular}

TE, Tissue Expension; AFT, Autogolous Fat Transfer; The unit of the size of TE, the amount of NS and the volume of AFT is ml. 

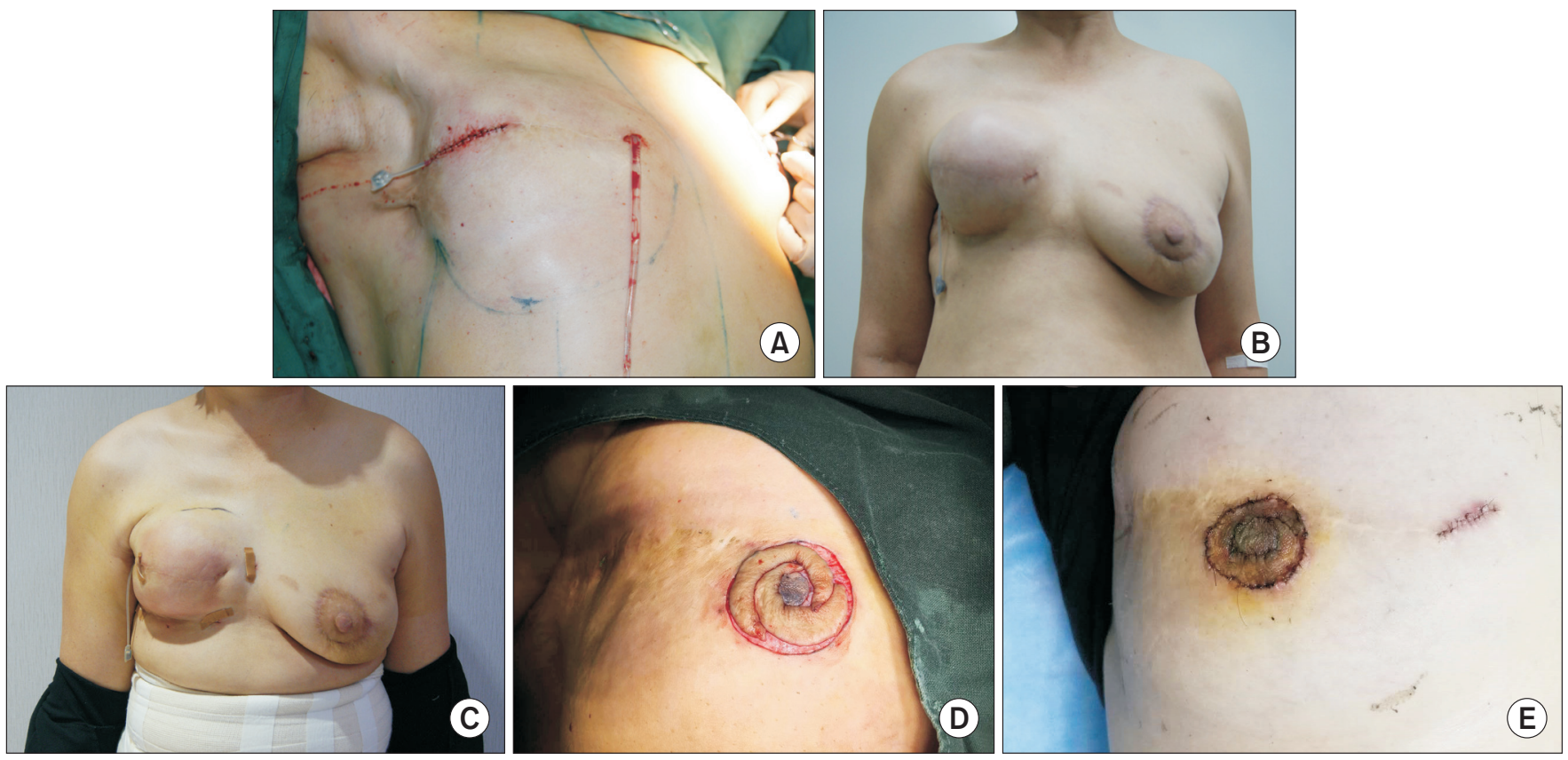

Fig. 1. (A) The expander, filled with $300 \mathrm{ml}$ antibiotic saline, was placed beneath the pectoralis major, generally without any skin tension. The injecting port was pulled out of the pocket through the incision at the mastectomy draining scar and kept out of the body until the expander was removed. (B) Five months after the placement of tissue expander, the patient was ready for the first AFT. (C) A day after the first AFT. (D) The full thickness nipple and areola were ready for free graft on the reconstructed breast. (E) The reconstructed nipple-areola complex on the reconstructed breast 10 days postoperatively.

peripheral part of the contralateral areola was removed in full thickness, and the donor site was closed primarily. If the diameter of the areola was $<4 \mathrm{~cm}$, the donor site would be the minor labia, or a tattoo was used to mimic the contralateral areola. In the recipient site, a 3.5-cm-diameter circle was marked on the symmetrical site of the contralateral NAC, and the skin was removed deep to the subdermal layer. Subsequently, the nipple and areola or minor labia were transferred to the recipient site as free grafts. Slight touch-ups were performed by autologous transfer of a small amount of fat (Fig. 1D, Fig. 1E).

\section{Case report}

A 62-year-old Chinese woman was admitted to our division for delayed right breast reconstruction. Ten years prior, the patient underwent mastectomy of the right breast because of breast cancer (IIb). The patient underwent chemotherapy after the first oncological surgery. However, she did not undergo radiation of the breast. The patient did not prefer TRAM and DIEP flap tor breast implants as breast reconstructive method because of the risk of secondary injury of the donor site and a reaction to a foreign biomaterial in her body. We introduced her to Brava for poor chest condition owing to mastectomy. How- ever, the patient could not afford Brava. Hence, replacing Brava with tissue expander followed by serial AFT was the only option. The patient opted for our plan after being informed of the possibility of failure, the loss of all fat graft. The treatment was performed using the abovementioned methods. All treatments were completed after more than 2 years, and the patient had her breast reconstructed with a very satisfactory result (Table 1 , Fig. 2).

\section{Results}

All six patients had satisfactory results. One patient underwent three AFTs, whereas one patient and four patients underwent four and five AFTs, respectively. Two adjacent AFTs were performed at 3-5-month intervals because the patients did not return for their next scheduled treatment. In this series, $450 \mathrm{ml}$ round expanders were used, and the volume of each AFT was 50-150 ml. Two patients underwent mastopexy on the contralateral breasts to correct severe breast ptosis. One patient underwent expander insertion during the first surgery, and the another patient underwent slight touch-up of the reconstructed breast during the last surgery. Two patients underwent NAC reconstruction 6 months after the last AFT. 

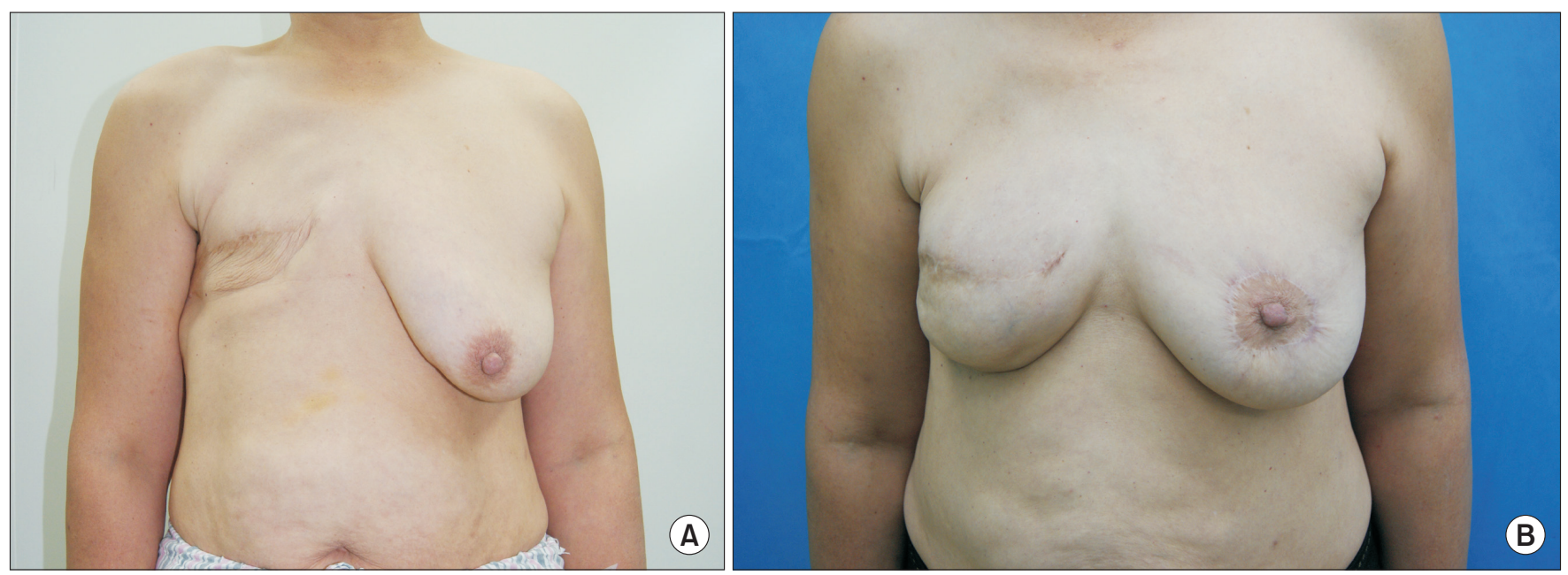

Fig. 2. All treatments were completed more than 2 years, and the patient finally underwent breast reconstruction, with a very satisfactory result. A. preoperative B. postoperative.

\section{Discussion}

Clinical experiences showed that using the Brava external expansion system for the enhancement of fat grafting is a suitable technique for breast reconstruction after mastectomy. Furthermore, the technique produces soft and natural-looking breasts, with fewer surgical sessions and minimal risk of complications [4]. However, the Brava system still has unique complications, such as erythema, pruritus, and skin lesions $[7,8]$. Some patients in developing countries cannot afford Brava. Hence, replacing Brava with a tissue expander can be still considered an option for patients who may be allergic to or cannot afford Brava.

The findings of this case indicated that the fat graft can take in the expanded flap. However, because tissue expansion belongs to the internal expansion system, it is completely different from Brava's external expansion system. The latter creates a larger and more fertile recipient matrix that will allow more fat graft droplets to be diffusely dispersed, with each fat graft droplet maintaining the crucial graft-to-recipient interface contact required for revascularization [3]. The former, however, creates more skin area, whereas the subcutaneous tissue and even the pectoralis major may be pressed and become flattened compared to that of the preoperative condition. When the expander is partly deflated during AFT, the pressure in the subcutaneous tissue decreases, which will benefit to effusion of the tissue fluid and the take of the grafted fat. Furthermore, the adipose tissue is rich in stem cells and therefore has a great capacity for angiogenesis [1]. This property and the increase in vascularization will improve the tissue quality and support serial fat grafting into the expanded soft tissue.
Symmetrical breast reconstruction to the contralateral site is challenging when the normal breast has ptosis. This situation can be resolved by performing mastopexy of the normal side or lowering the inframammary fold of the reconstructed side. Because the expanded tissue and skin have different mechanical strengths and pliabilities from the nearby tissue, suitable lowering of the inferior margin of the undermined area will make the newly reconstructed breast similar to the contralateral breast. However, the extent of dissection depends on the degree of ptosis in the contralateral breast. If mild and moderate or severe breast ptoses were noted, the lower margin of the expander pocket should be $2-3 \mathrm{~cm}$ and $3-4 \mathrm{~cm}$ lower than the contralateral inframammary fold, respectively.

Because of the successful breast mount reconstructed, two patients returned for NAC reconstruction. The NAC donor was from the contralateral NAC. The nipple of the normal side was cut in half from its center. In one patient, the peripheral part of the contralateral areola was removed in full thickness as the donor of the areola. However, in another patient, the diameter of the areola was $<4 \mathrm{~cm}$; hence, a tattoo was used to mimic the contralateral areola.

In this series, we found that breast reconstruction with tissue expansion followed by serial AFT is feasible. It can be an option for patients who do not prefer pedicled TRAM or DIEP flap transfer or pedicled latissimus dorsi myocutaneous flap coverage with implant placement and who cannot afford Brava plus fat graft. However, we cannot generalize the validity of the surgery because the patient sample may be too small. Thus, studies involving a large sample and further experiments are still needed in the future. The disadvantage of this method is 
performing the surgery 4-5 times. Decreasing the number of patient visits and increasing the amount of graft will be our next attempt.

\section{Conflicts of interest}

The authors have nothing to disclose.

\section{References}

1. Coleman SR, Saboeiro AP. Fat grafting to the breast revisited: safety and efficacy. Plast Reconstr Surg 2007;119:775-85; discussion 786-7.

2. Curtin C. Breast reconstruction. In: Brown DL, Borschel GH, editors. Michigan manual of plastic surgery. Philadelphia: Lippincott Williams \& Wilkins; 2004. p. 242-7.

3. Khouri RK, Eisenmann-Klein M, Cardoso E, Cooley BC, Kacher D, Gombos E, et al. Brava and autologous fat transfer is a safe and effective breast augmentation alternative: results of a 6-year, 81-patient, prospective multicenter study. Plast Reconstr Surg 2012;129:1173-87.

4. Mestak O, Mestak J, Bohac M, Edriss A, Sukop A. Breast reconstruction after a bilateral mastectomy using the BRAVA expansion system and fat grafting. Plast Reconstr Surg Glob Open 2013;1:e71.

5. Khouri RK, Rigotti G, Khouri RK Jr, Cardoso E, Marchi A, Rotemberg SC, et al. Tissue-engineered breast reconstruction with Brava-assisted fat grafting: a 7-year, 488-patient, multicenter experience. Plast Reconstr Surg 2015;135:643-58.

6. Hammer-Hansen N, Jensen TB, Damsgaard TE. Delayed total breast reconstruction with brava. Case Rep Surg 2015;2015:601904.

7. Uda H, Sugawara Y, Sarukawa S, Sunaga A. Brava and autologous fat grafting for breast reconstruction after cancer surgery. Plast Reconstr Surg 2014;133:203-13.

8. Ho Quoc C, Delay E. Tolerance of pre-expansion BRAVA and fat grafting into the breast. Ann Chir Plast Esthet 2013;58:21621. 